\title{
Nosocomial infection with SARS-CoV-2 and main outcomes after surgery within an orthopaedic surgery department in a tertiary trauma centre in Spain
}

\author{
Kushal Lakhani $^{1,2}$ (D) Joan Minguell ${ }^{1,2} \cdot$ Ernesto Guerra-Farfán $^{1,2} \cdot$ Yuri Lara $^{1,2} \cdot$ Unai Jambrina $^{1,2} \cdot$ Joan Pijoan $^{1,2} \cdot$ \\ Jorge H. Núñez ${ }^{1,2}$
}

Received: 2 July 2020 / Accepted: 1 September 2020 / Published online: 11 September 2020

(C) SICOT aisbl 2020

\begin{abstract}
Aims The purpose of the present study is to analyse clinical data of a series of cases who developed nosocomial infection with SARS-CoV-2 in an orthopaedic and traumatology department.

Patients and methods In this non-interventional retrospective study, carried out at a tertiary hospital within the Spanish National Health System, all adult patients who were admitted in the Orthopaedic Surgery and Traumatology Department between March 9th and May 4th, 2020, were included. Clinical, biological and radiological data, as well as mortality rates, were collected from hospital medical records.

Results A total of 293 periods of hospitalization were analysed in 288 patients. Mean age was 66.1 years old and $57.3 \%$ were females. Nineteen patients $(6.48 \%)$ met the inclusion criteria to be categorized as a nosocomial infection with SARS-CoV-2. In a comparison between patients with and without nosocomial infection, age, mortality and hospital length of stay were statistically significant $(p<0.05)$. The median time from admission to diagnosis of SARS-CoV-2 infection in our cohort was 16 days $(6-$ 86 days). No statistically significant differences were found in sex, living situation, reason of admission or period of admission (even if we observed that most of the nosocomial infections (78.9\%) occurred in March).

Conclusion We have found a $6.48 \%$ of nosocomial infection with SARS-CoV-2, but with an important reduction of it after undergoing preventing protocols that included screening RT-PCR test for COVID-19. Age and hospital length stay were statistically significant risk factors for nosocomial infection with SARS-CoV-2. For the progressive restoration of the surgical activity, we recommend to correctly select the patients in elective surgery and to encourage fast-track programs and early discharge of patients with fractures.
\end{abstract}

Keywords Nosocomial infection $\cdot$ SARS-CoV-2 $\cdot$ Coronavirus · COVID-19 · Surgery · Orthopaedic

\section{Introduction}

On January 20th, 2020, the World Health Organization (WHO) declared a public health emergency due to a new coronavirus disease: severe acute respiratory syndrome coronavirus 2 (SARS-CoV-2). This coronavirus disease 2019

Kushal Lakhani

klakhani@vhebron.net; kushallakhani@hotmail.com

1 Department of Traumatology and Orthopedic Surgery, University Hospital of Vall d'Hebron, Passeig de la Vall d'Hebron, 119-129 08035 Barcelona Spain

2 Universitat Autónoma de Barcelona, Passeig de la Vall d'Hebron, 119-129 08035 Barcelona Spain
(COVID-19) was classified as a pandemic on March 11th, 2020. Spain was one of the first European countries to deal with this virus outbreak, declaring the State of Alarm on March 14th, 2020. This State of Alarm had several consequences on the Spanish population, imposing restrictions mainly in the free circulation of people and educational activity. People were allowed to move around public spaces only in very specific cases, like buying food or medicines, and all educational activities were cancelled [1].

Hospitals were forced to adapt their sources to face the impact of the virus, reducing the surgical activity to the minimum. In our hospital, all elective-surgical activity was suspended from March 16th, 2020, and only traumatic and nondelayable septic or oncological surgery was taken. However, as one of the trauma care centre referrals in Spain, covering a 
catchment area of more than 550,000 inhabitants, medical assistance was needed to supply to a considerable number of patients in this period. Special measures were adopted to minimize the risk of infection with COVID-19 for patients as well as for health professionals. Notwithstanding protective measures, the risk of contracting the virus during hospitalization period (or immediate outpatient care) did not appear to be unimportant, and the main clinical outcomes of the patients, such as early mortality, seemed to be magnified.

Very few data about nosocomial infection with SARSCoV-2 is available in the literature [2]. This parameter seems to be a key factor for the progressive restoration of normal surgical activity in hospitals. Thus, the purpose of the present study is to analyse clinical data of a series of cases who developed nosocomial infection with SARS-CoV-2 in an orthopaedic and traumatology department.

\section{Patients and methods}

\section{Study design and participants}

This is a non-interventional retrospective study, carried out at a tertiary hospital within the Spanish National Health System. All adult patients who were admitted in the Orthopaedic Surgery and Traumatology Department between March 9th and May 4th, 2020, were included. This period was selected as it included the immediately preceding week of the first patient diagnosed with SARS-CoV-2 in our department, which took place on March 15th, 2020. Exclusion criteria included patients under the age of 16 , as these patients were admitted in a different building of our hospital. From March 9th, 2020, to May 4th, 2020 , a total of 293 periods of hospitalization were analysed in 288 patients. Mean age was 66.1 years old, standard deviation (SD) $20.8 ; 57.3 \%$ were females. Characteristics of the patients are reported on Table 1. Reason for admission in our department is reported in Fig. 1, and all orthopaedic procedures done are analysed in Fig. 2.

Data about age, gender, living situation, diagnosis, date of admission and discharge, dates and types of surgery performed, status of COVID-19's screening, data of ulterior positive test and early mortality were collected from hospital medical records. If the same patient was hospitalized twice, each hospitalization was counted as a different period. Likewise, for patient who developed nosocomial infection with SARS-CoV-2, data about comorbidities, nutritional status, diagnose contextualization, clinical course and medical and surgical complications were further investigated. Clinical outcomes for all patients were monitored up to May 18th, 2020. At the moment of recollecting data, eight patients were still hospitalized. Ethical approval for this study was obtained from the Research Ethics Committee of our institution (PR(AG)336/2020).
Table 1 Description of characteristics of all patients analysed (from March 9th to May 4th, 2020)

\begin{tabular}{ll}
\hline Characteristics & Periods of hospitalization $(n=293)$ \\
\hline Age (years) & $66.1(20.8)$ \\
Mean (SD) & \\
Gender \% (n) & $42.7(125)$ \\
Male & $57.3(168)$ \\
Female & \\
Residency \% (n) & $91.1(267)$ \\
Family home & $5.4(16)$ \\
Nursing home & $3.4(10)$ \\
Transfer from other hospital & \\
Status before surgery \% (n) & $61.8(181)$ \\
Hospitalized & $38.2(112)$ \\
Non-hospitalized & $4.9(14)$ \\
Emergency surgeries \% $(n)$ & $8(3-15)$ \\
Hospital length of stay (days) & \\
Median (interquartile range) & \\
COVID-19 screening test \% $(n)$ & $3.4(10)$ \\
Positive & $31.4(92)$ \\
Negative & $65.2(191)$ \\
Not performed & $90.1(264)$ \\
Mortality \% $(n)$ & \\
No & \\
Yes & \\
\hline
\end{tabular}

$\%$ percentage, $n$ : number, $S D$ : standard deviation

\section{Nosocomial infection with SARS-CoV-2}

Definition of nosocomial infection with SARS-CoV-2 was based on current studies that have tried to investigate the length of the incubation period of COVID-19 [3, 4]. Lauer et al. estimated the median incubation period to be 5.1 days (CI 4.5-5.8) and underlined that after 14 days, it is highly unlike that further symptomatic infections would be undetected among high-risk individuals, as is the case of recent

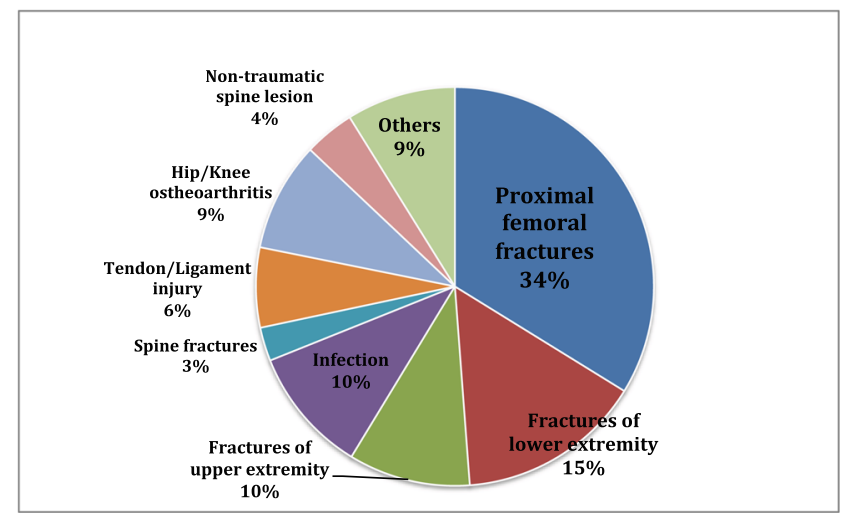

Fig. 1 Reason for admission of all patients 


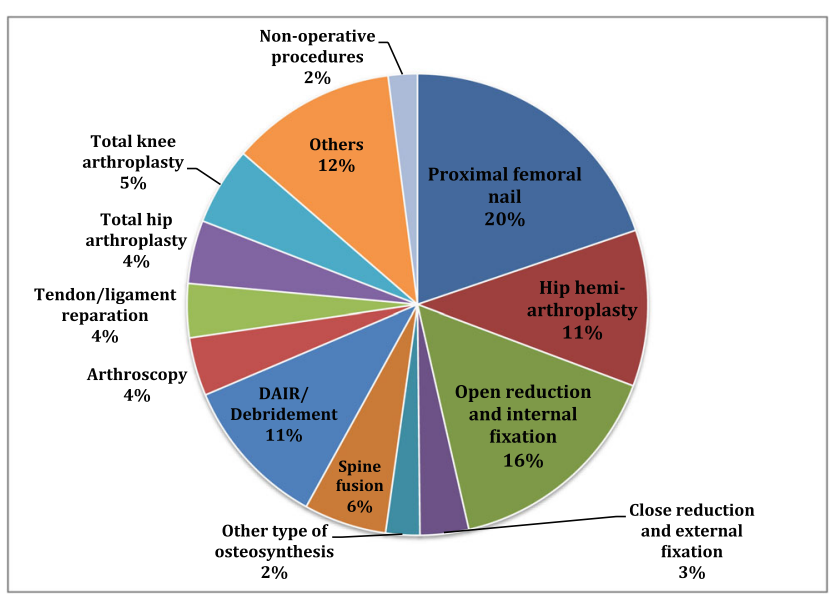

Fig. 2 Orthopaedic procedures of all patients. Other procedures (12\%) mainly included hardware removal, peripheral nerve revision surgery, closed reduction and cast application, joint infiltration (hip and thoracic and lumbar facet joint injection) and correction surgery of various foot deformities

hospitalized patients [4]. Thus, in our study, nosocomial infection with SARS-CoV-2 was defined by meeting these requirements:

- Not having a positive test screening at admission or clinical symptoms suggestive of SARS-CoV-2 infection.

- Developing COVID-19 infection during the hospitalization period or during the first 14 days after discharge.

If screening test was not performed, patient was categorized as developing nosocomial infection with SARS-CoV-2 if the diagnosis was done after the fourth day of hospitalization, according Lauer et al. investigations [4].

\section{Protective measures}

External consultations were converted to telematics phonecall consultation whereas possible. Additionally, systematic screening with thorax X-Ray, nasopharyngeal swabs and a quantitative PCR test to detect SARS-CoV-2 infection of patients upon admission into our department was implanted on March 30th, 2020. Until then, the test was performed only in case of suggestive symptoms or recent contact with a COVID-19+ patient. Droplets and contact isolation precautions were adopted in all patients having a positive PCR test, until negative result. All chest X-Ray were reviewed and evaluated by a radiologist. Emergency department was also divided into two different circuits to separate COVID$19+$ patients and non-COVID-19 patients. Social distancing was applied among healthcare workers when possible, including common spaces as dining halls, lifts and work spaces. Additionally, family visits were also prohibited from March 16th, 2020, onward.

\section{Statistical analysis}

Categorical variables were presented with their absolute values and percentages and quantitative variables were expressed as mean (standard deviation, SD). Continuous variables were compared using Student's $t$ test or Mann-Whitney $U$ test, whereas categorical variables were compared using the $\chi^{2}$ test or Fisher's exact test, as appropriate. The most relevant risk factors associated with nosocomial SARS-Cov-2 infection were selected in the bivariate logistic regression analysis. Variables with a $p$ value $<0.05$ in the bivariate logistic regression analysis were included in the final multivariate logistic regression analysis. A $p$ value $<0.05$ was considered statistically significant. SPSS software (version 23.0; IBM) was used for statistical analysis of collected data.

\section{Results}

During this period, from the 52 staff members and 26 residents of the Orthopaedic Team, eight members (4 staff members and 4 residents) were diagnosed with COVID-19 infection. Nineteen patients $(6.48 \%)$ met the inclusion criteria to be categorized as a nosocomial infection with SARS-CoV-2 (Table 2). Statistically significant differences were found in age, sex, residence, reasons for admission, mortality, period of admission and length of hospital stay $(p<0.05)$ between patients not infected with COVID-19, patients with nosocomial infection and patients infected at the time of admission (Table 3). From the patients who had a positive test result at admission, mean age was higher comparing with the other two groups (mean age 83.3 years old; SD 6.7). Furthermore, $100 \%$ of these patients were women, percentage who lived in nursing home was higher (40\%) and mortality was also superior $(50 \%)$.

Analysing the reason for admission of the nineteen patients who developed nosocomial infection with SARS-CoV-2 according to our definition, incidence in each subgroup of patients susceptible to develop it was calculated. A total of $11 \%$ of the susceptible patients with a proximal femoral fracture acquired it, as the $7 \%$ of the patients with a lower extremity fracture and the $13.8 \%$ of the patients with an infection-related process developed it too. None of the 26 patients hospitalized during the study period who underwent a THR or TKR developed a nosocomial infection with SARS-CoV-2.

Evaluating the medical and surgical complications after surgery of the 19 mentioned patients, only two patients were registered with no complications. Eight patients developed severe anaemia according to WHO classification $(\mathrm{Hb}<$ $8.0 \mathrm{~g} / \mathrm{dL}$ ), seven suffered acute respiratory failure (one of them due to an acute pulmonary oedema) and six developed cardiac failure ( 2 of them due to a new onset of atrial fibrillation). Additionally, two patients developed urinary tract infection 
Table 2 Description of all characteristics, including laboratory data at the time of diagnosis, of the patients developing nosocomial infection with COVID-19

Clinical characteristics

Patients developing nosocomial infection with COVID-19 $(n=19)$

Age (years)

Mean (SD)

$78.3(19.9)$

Gender \% (n)

Male

$47.4(9)$

Female

$52.6(10)$

Residency \% (n)

Family home

$84.1(16)$

Nurse home

$10.6(2)$

Transfer from other hospital

$5.3(1)$

Comorbidities \% (n)

Smoking history

$31.6(6)$

Hypertension

68.4 (13)

COPD

$5.3(1)$

Asthma

$15.8(3)$

Cerebrovascular accident

$21.1(4)$

Ischemic cardiopathy

$21.1(4)$

Dyslipidaemia

$26.3(5)$

Chronic renal failure

15.8 (3)

Cancer history

$26.3(5)$

Reason for admission \% ( $n)$

Proximal femoral fracture

$52.6(10)$

Fracture of lower extremity

15.8 (3)

Infection

$21.1(4)$

Polytrauma

$5.3(1)$

Non-traumatic spine lesion

$5.3(1)$

Hospital length of stay before COVID-19 diagnosis (days)

Median (range)

$16(6-86)$

COVID-19 screening test $\%(n)$

Negative

$36.8(7)$

Not performed

$63.2(12)$

Moment of COVID-19 diagnosis \% (n)

During hospitalization

$84.2(16)$

After hospital discharge

$15.8(3)$

Do-Not-Resuscitate status before COVID-19 diagnosis \% ( $n$ )

$52.6(10)$

Signs and symptoms at the time of diagnosis \% (n)

Cough

$63.2(12)$

Fever

$47.4(9)$

Dyspnoea

$21(4)$

Diarrhoea

$0(0)$

Oxygen saturation on room air at the time of diagnosis

$\%$ (range)

$95(82-99)$

Most aggressive ventilation mode applied \% (n)

Room air

$47.4(9)$

Nasal cannulae

$5.3(1)$

High-concentration oxygen mask

$42.1(8)$

Tracheostomy

$5.3(1)$

Albumin (g/dL)

Mean (SD) 
Table 2 (continued)

\begin{tabular}{ll} 
Clinical characteristics & Patients developing nosocomial infection with COVID-19 $(n=19)$ \\
\hline Leukocytes count $\left(10^{9} / \mathrm{L}\right)^{*}$ & $7.22(3.02-14.86)$ \\
Neutrophils $\left(10^{9} / \mathrm{L}\right)^{*}$ & $5.2(1.6-13)$ \\
Lymphocytes $\left(10^{9} / \mathrm{L}\right)^{*}$ & $1(0.1-2.7)$ \\
C-reactive protein $(\mathrm{mg} / \mathrm{L})^{*}$ & $6.2(1.87-18.7)$ \\
Interleukin-6* & $37.06(1.89-1196)$ \\
Ferritin* & $770(352-3118)$ \\
D-dimer* & $1335(300-11,000)$ \\
Mortality \% $(n)$ & \\
No & $63.2(12)$ \\
Yes & $36.8(7)$ \\
\hline
\end{tabular}

$\%$ percentage, $n$ number, $S D$ standard deviation, $C O P D$ chronic obstructive pulmonary disease

*Values are given as the median (range)

with Escherichia coli; one patient suffered of a deep venous thrombosis of the upper extremity. Only one surgical complication was registered: a wound infection after spinal fusion in a 44-year-old polytrauma patient, who required a surgical debridement.

In a comparison between patients with and without nosocomial infection, only age, mortality and hospital length of stay were statistically significant $(p<0.05)$. No statistically significant differences were found in sex $(p=0.77)$, living situation $(p=0.31)$, reason of admission $(p=0.19)$ or period of admission $(p=0.15)$ (Table 4$)$. Using the bivariate and multivariate logistic regression to assess risk factors of nosocomial COVID-19 infections, age (Odds ratio (OR) 16.5; $95 \%$ confident interval (CI): 68.9-87.5 years; $p<0.007)$ and hospital length of stay (OR 7.6; 95\% CI: 23.5-39.7 days; $p<0.001$ ) were statistically significant (Table 5). Additionally, all nosocomial COVID-19 infections occurred when there were admitted more than 450 patients with COVID-19 infection in our institution. However, there was no statistical significance on this issue $(p=0.99)$ (Fig. 3). On April 1st, 2020, over 800 patients were hospitalized in our institution with COVID-19 infection. Our hospital was classified thereby as the hospital in Catalonia that attended the highest amount of patients with COVID-19 infection during the outbreak (over 2000 patients).

\section{Discussion}

The risk of nosocomial infection with SARS-CoV-2 seems to be a key factor for the progressive restoration of normal surgical activity in hospitals. In our study, we had a $6.48 \%$ (19/ 293 ) of nosocomial infection with SARS-CoV-2. There was a higher mortality in patients admitted with COVID-19 (50\%) and patients with nosocomial infection (36.8\%) comparing with patients without COVID-19 infection (6.4\%). The principal findings of our study were that age (OR 16.5; 95\% CI: 68.9-87.5 years; $p<0.007)$ and hospital length of stay (OR 7.6; 95\% CI: 23.5-39.7 days; $p<0.001)$ were statistically significant risk factor for developing nosocomial infection with SARS-CoV-2.

Minimizing nosocomial transmission of COVID-19 remains a challenge, given the wide spectrum of respiratory syndromes and mild respiratory symptoms at presentation $[5,6]$. Nosocomial transmission has emerged as a significant concern; in Wuhan, almost one-third of an initial cohort of COVID-19 patients were healthcare workers (HCWs) and hospitalized patients [7]. To our knowledge, only two studies have reported the rate of nosocomial infection with SARSCoV-2 between patients. Luong-Nguyen et al. reported a $4.9 \%(15 / 305)$ of nosocomial infection with SARS-CoV-2 in three different department of digestive surgery [2]. Vanhems et al. reported an infection rate of $20 \%$ among patients in a French geriatric unit [8]. Differences of these rates may be explained by the mean age of the patients analysed. Protective measures applied and early PCR test screening instauration may have also played an essential role to decrease nosocomial infection with COVID-19. In Luong-Nguyen et al., mean age of patients with COVID-19 nosocomial infection was 62 years old; meanwhile, in our study, the mean age was 78.3 years old. Vanhems et al. reported that the index case had 97 years old.

We found that age was a statistically significant risk factor for nosocomial infection with SARS-CoV-2 $(p<0.007)$. In our study, the mean age of all patients was 66.1 years old (range 16-101). This high mean age may be explained because of the demographics of Spain, as the $18.15 \%$ of the Spanish population are 65 years old or older. Spain is currently expected to be the country with the most elderly population in the world by 2040 
[9]. As it has been already reported, despite the contingency plans and lockdown measures, the incidence of osteoporotic hip fractures seemed to remain stable during the State of Emergency (implementation of stringent lockdown measures for the population) $[10,11]$. In the present study, proximal femoral fracture was the most common reason for admission $(33.8 \%)$. As the increase in the severity of COVID-19 with age is clearly studied [12], protective measurements were applied universally in our department, but with special emphasis in elderlies.

We observed that most of the nosocomial infections occurred in March (78.9\%), when the outbreak was higher. Until March 31st, at admission, an RT-PCR for COVID19 was only requested if the patients had symptoms or had a pathological chest X-ray evaluated by a radiologist (Fig. 4). Due to the increase of pandemic outbreak and looking after the protection of health workers, the protocol was improved. All patients admitted wore surgical mask and underwent an RT-PCR test for COVID-19. The positive effects of these measures were studied with the bivariate regression analyses, which did not find statistically significant differences between the patients hospitalized with COVID-19 in our institution and the risk of developing nosocomial SARS-CoV-2 infection in our department (OR 1.0; 95\% CI: $0.998-1.002 ; p=0.80$ ).

The median time from admission to diagnosis of SARSCoV-2 infection in our cohort was 16 days (range of 686 days). Hospital length of stay was a statistically significant risk factor for nosocomial infection with SARS-CoV-2 $(p<$ 0.001). Luong-Nguyen et al. reported a median time from admission to diagnosis of SARS-CoV-2 infection of 34 days (5-61 days). In their study, 12 patients (80\%) were diagnosed

Table 3 Description of general characteristics of patients (analysed by groups, depending on the COVID-19 infection status)

\begin{tabular}{|c|c|c|c|c|}
\hline & \multicolumn{4}{|l|}{ COVID-19 infection } \\
\hline & Non-infected $(n=264)$ & Nosocomial infection $(n=19)$ & Infected at admission $(n=10)$ & $p$ value \\
\hline \multicolumn{5}{|l|}{ Age (years) } \\
\hline Mean (SD) & $64.5(20.7)$ & $78.3(19.6)$ & $83.3(6.8)$ & $<0.05$ \\
\hline \multicolumn{5}{|l|}{ Gender \% (n) } \\
\hline Male & $43.9(116)$ & $47.4(9)$ & $0(0)$ & \multirow[t]{2}{*}{0.02} \\
\hline Female & $56.1(148)$ & $52.6(10)$ & $100(10)$ & \\
\hline \multicolumn{5}{|l|}{ Residency \% (n) } \\
\hline Family home & $93.2(246)$ & $84.1(16)$ & $50(5)$ & \multirow[t]{3}{*}{0.001} \\
\hline Nursing home & $3.8(10)$ & $10.6(2)$ & $40(4)$ & \\
\hline Transfer from other hospital & $3.0(8)$ & $5.3(1)$ & $10(1)$ & \\
\hline \multicolumn{5}{|l|}{ Reason for admission $\%(n)$} \\
\hline Proximal femoral fracture & $30.7(81)$ & $52.6(10)$ & $80(8)$ & \multirow[t]{5}{*}{0.001} \\
\hline Fracture of lower extremity* & $15.2(40)$ & $15.8(3)$ & $10(1)$ & \\
\hline Fracture of upper extremity & $11.0(29)$ & $0(0)$ & $0(0)$ & \\
\hline Infections & $9.5(25)$ & $21.1(4)$ & $10(1)$ & \\
\hline Others & $33.6(89)$ & $10.5(2)$ & $0(0)$ & \\
\hline \multicolumn{5}{|l|}{ Hospital length of stay (days) } \\
\hline Mean (SD) & $11.2(16.9)$ & $31.7(28.9)$ & $17.7(12.8)$ & $<0.05$ \\
\hline \multicolumn{5}{|l|}{ Mortality \% (n) } \\
\hline No & $93.6(247)$ & $63.2(12)$ & $50.0(5)$ & \multirow[t]{2}{*}{0.001} \\
\hline Yes & $6.4(17)$ & $36.8(7)$ & $50.0(5)$ & \\
\hline \multicolumn{5}{|l|}{ Period of admission \% ( $n)$} \\
\hline Before 1st March & $10.6(28)$ & $21.1(4)$ & $0(0)$ & \multirow[t]{6}{*}{0.002} \\
\hline Between 1st and 15th March & $45.1(119)$ & $36.8(7)$ & $0(0)$ & \\
\hline Between 16th and 31st March & $14.0(37)$ & $31.6(6)$ & $0(0)$ & \\
\hline Between 1st and 15th April & $13.3(35)$ & $5.3(1)$ & $60(6)$ & \\
\hline Between 16th and 30th April & $16.7(44)$ & $5.3(1)$ & $30(3)$ & \\
\hline After 30th April & $0.3(1)$ & $0(0)$ & $10(1)$ & \\
\hline
\end{tabular}

$\%$ percentage, $n$ number, $S D$ standard deviation

*Does not include proximal femoral fractures 
Table 4 Description of general characteristics of patients (analysed by groups, depending on the COVID-19 infection status and excluding patients with COVID-19 infection at admission)

\begin{tabular}{|c|c|c|c|}
\hline & \multicolumn{3}{|c|}{ Nosocomial COVID-19 infection } \\
\hline & No $(n=264)$ & Yes $(n=19)$ & $p$ value \\
\hline \multicolumn{4}{|l|}{ Age (years) } \\
\hline Mean (SD) & $64.5(20.7)$ & $78.3(19.6)$ & $<0.05$ \\
\hline \multicolumn{4}{|l|}{ Gender \% (n) } \\
\hline Male & $43.9(116)$ & $47.4(9)$ & \multirow[t]{2}{*}{0.85} \\
\hline Female & $56.1(148)$ & $52.6(10)$ & \\
\hline \multicolumn{4}{|l|}{ Residency \% ( $n$ ) } \\
\hline Family home & $93.2(246)$ & $84.1(16)$ & \multirow[t]{3}{*}{0.31} \\
\hline Nursing home & $3.8(10)$ & $10.6(2)$ & \\
\hline Transfer from other hospital & $3.0(8)$ & $5.3(1)$ & \\
\hline \multicolumn{4}{|l|}{ Reason for Admission \% $(n)$} \\
\hline Proximal femoral fracture & $30.7(81)$ & $52.6(10)$ & \multirow[t]{5}{*}{0.19} \\
\hline Fracture of lower extremity* & $15.2(40)$ & $15.8(3)$ & \\
\hline Fracture of upper extremity & $11.0(29)$ & $0(0)$ & \\
\hline Infections & $9.5(25)$ & $21.1(4)$ & \\
\hline Others & $33.6(89)$ & $10.5(2)$ & \\
\hline \multicolumn{4}{|l|}{ Hospital length of stay (days) } \\
\hline Mean (SD) & $11.2(16.9)$ & $31.7(28.9)$ & $<0.05$ \\
\hline \multicolumn{4}{|l|}{ Mortality \% (n) } \\
\hline No & $93.6(247)$ & $63.2(12)$ & \multirow[t]{2}{*}{0.001} \\
\hline Yes & $6.4(17)$ & $36.8(7)$ & \\
\hline \multicolumn{4}{|l|}{ Period of admission \% ( $n)$} \\
\hline Before 1st March & $10.6(28)$ & $21.1(4)$ & \multirow[t]{6}{*}{0.15} \\
\hline Between 1st and 15th March & $45.1(119)$ & $36.8(7)$ & \\
\hline Between 16th and 31st March & $14.0(37)$ & $31.6(6)$ & \\
\hline Between 1st and 15th April & $13.3(35)$ & $5.3(1)$ & \\
\hline Between 16th and 30th April & $16.7(44)$ & $5.3(1)$ & \\
\hline After 30th April & $0.3(1)$ & $0(0)$ & \\
\hline
\end{tabular}

$\%$ percentage, $n$ number, $S D$ standard deviation

*Does not include proximal femoral fractures

after a hospital length of stay greater than 14 days [2]. However, they only made a descriptive analysis of patients with nosocomial SARS-CoV-2 infection, with no comparison [2]. Due to the imminent restoration of the surgical activity, to understand modifiable risk factors of nosocomial SARS-CoV2 infection becomes crucial for all surgery departments. As most of orthopaedic surgery is elective, pre-operative optimization of patients is more essential than ever [13]. Modifiable variables and correct selection of patients, as well as preventing protocols to restrict increases of hospital length stay, should be carefully evaluated. In a systematic review and meta-analysis of 21,494,459 patients undergoing a knee arthroplasty, Shah et al. found that a pre-operative haemoglobin $<13.0 \mathrm{~g} / \mathrm{dL}(95 \% \mathrm{CI}=0.34$ to $0.98 ; p<0.001)$
Table 5 Bivariate and multivariate analysis of the risk factors for nosocomial COVID19 infection

\begin{tabular}{llllll}
\hline & \multicolumn{3}{l}{ Bivariate } & & \multicolumn{2}{l}{ Multivariate } & \\
\cline { 2 - 3 } \cline { 5 - 6 } & OR $(95 \% \mathrm{CI})$ & $p$ value & & OR $(95 \% \mathrm{CI})$ & $p$ value \\
\hline Age & $1.06(1.02-1.10)$ & $p=0.02$ & & $16.5(68.9-87.5)$ & $p=0.007$ \\
Hospital length of stay & $1.04(1.02-1.16)$ & $p=0.001$ & & $7.6(23.5-39.7)$ & $p=0.001$ \\
\hline
\end{tabular}

$O R$ odds ratio, $C I$ confidence interval 


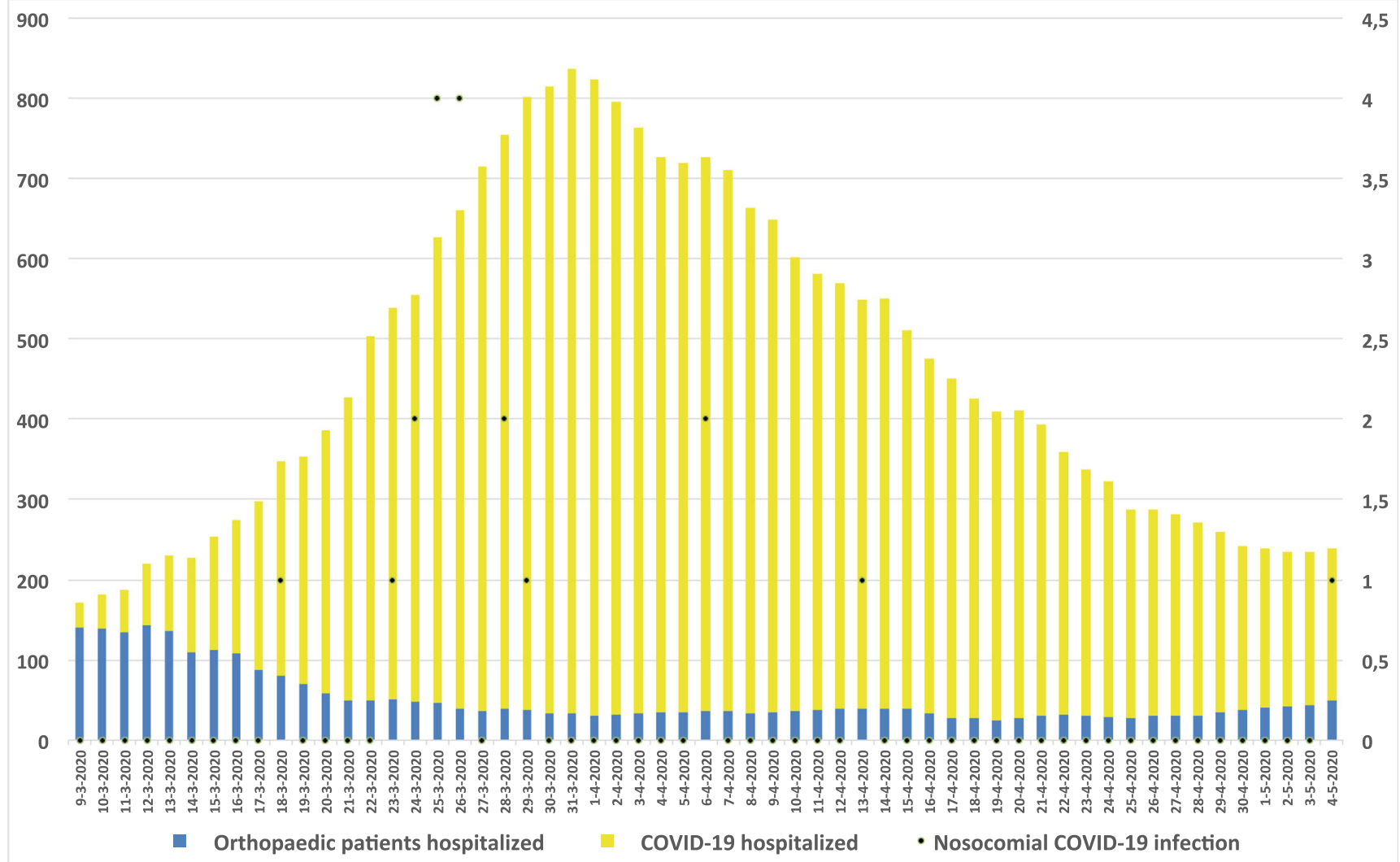

Fig. 3 Relation between number of COVID-19+ patients in our institution, number of patients hospitalized at our department and nosocomial COVID19 infection

was a risk factor for an increased hospital length of stay [14]. In a prospective study, Piuzzi et al. concluded that increased age, higher body mass index and greater Charlson Comorbidity Index were predictors of a longer hospital length of stay $(p<0.001)[15]$. Notwithstanding, most studies have found that early discharge is not an independent risk factor for major complications or readmissions following arthroplasty $[16,17]$. As well, programs for a rapid return to home in elderly patients who have undergone surgery for fractures have already demonstrated better results in terms of readmission rates, mobility and autonomy $[18,19]$.
Our study has the limitations and weaknesses of retrospective designs, and the fact that RT-PCR test for COVID-19 was not performed to all patients prior to hospital discharge may be reducing the rate of nosocomial infection by this way. However, a follow-up of 14 days after discharge to all patients was made, and all suspicious cases were tested with a RT-PCR for COVID-19. Three patients were added to our cohort of patients with nosocomial infection in the first 14 days after hospital discharge, maybe overestimating the rate of nosocomial infection. To our knowledge, this is the first study to
Fig. 4 a Thorax X-ray at admission of an 82-year-old patient admitted in our hospital. b Thorax X-ray of the same patient after 27 days of hospitalization, presenting multifocal and bilateral reticular interstitial opacities with lower lobes predominance and alveolar opacity in the right upper lobe. Right lower lobe atelectasis. No pleural effusion
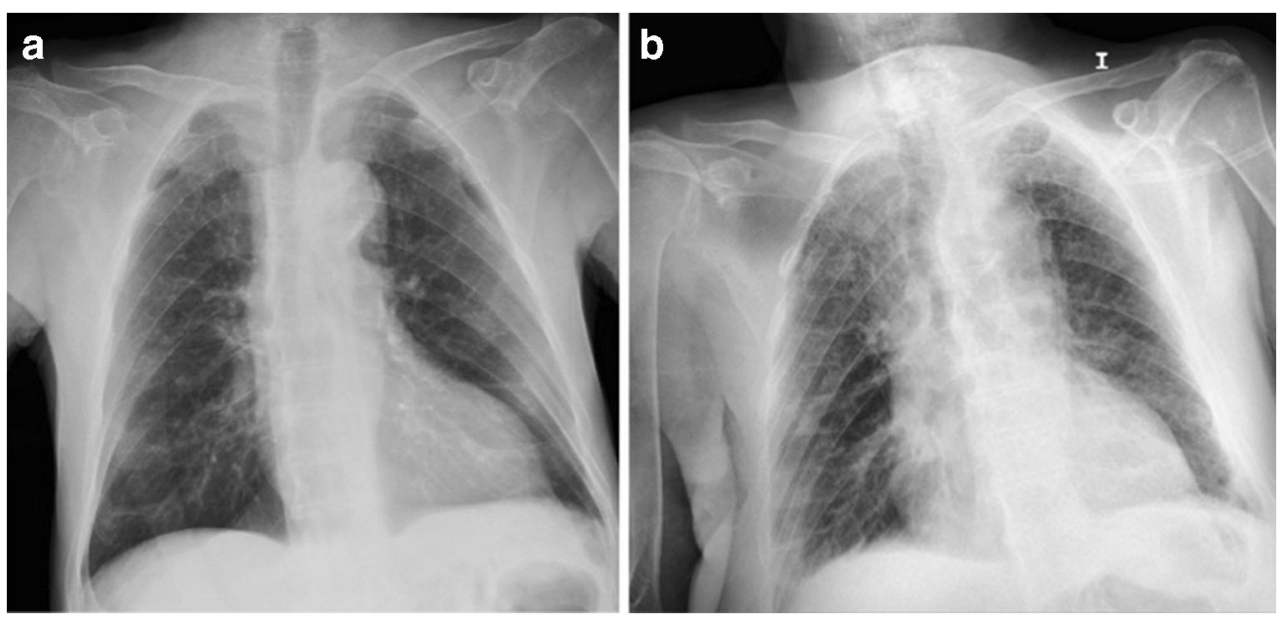
analyse risk factors of nosocomial infection with SARSCoV-2 within an orthopaedic surgery department.

To conclude, we have found in our study a $6.48 \%$ of nosocomial infection with SARS-CoV-2 between March 9th, 2020, and May 4th, 2020, but with an important reduction of it after undergoing preventing protocols that included screening RT-PCR test for COVID-19. Age and hospital length stay were statistically significant risk factors for nosocomial infection with SARS-CoV-2. For the progressive restoration of the surgical activity, we recommend to correctly select the patients in elective surgery and to encourage fasttrack programs and early discharge of patients with fractures.

Acknowledgments We would like to thank Dr. Magda Campíns-Martí, Head of Service of the Preventive Medicine and Public Health Department of the Hospital Universitari Vall d'Hebron (Barcelona, Spain), for her valuable work undertaken during the coronavirus outbreak and for the epidemiological assessment in conducting the present study.

Funding This research did not receive any specific grant from funding agencies in the public, commercial or not-for-profit sectors.

\section{Compliance with ethical standards}

Conflict of interest The authors declare that they have no conflict of interest.

\section{References}

1. Spanish Government (2020) Real Decreto 463/2020, de 14 de marzo, por el que se declara el estado de alarma para la gestión de la situación de crisis sanitaria ocasionada por el COVID-19. BOE $\mathrm{n}^{\mathrm{o}} 67$ de 14 de marzo de 2020. BOE. 2020;(14 de marzo): p. 25390-25400

2. Luong-Nguyen M, Hermand H, Abdalla S et al (2020) Nosocomial infection with SARS-Cov-2 within departments of digestive surgery. J Visc Surg 157(3):S13-S18

3. Vannabouathong C, Devji T, Ekhtiari S et al (2020) Novel coronavirus COVID-19. J Bone Jt Surg. 102(9):734-744

4. Lauer SA, Grantz KH, Bi Q et al (2020) The incubation period of coronavirus disease 2019 (CoVID-19) from publicly reported confirmed cases: estimation and application. Ann Intern Med 172(9): 577-582

5. Wee LE, Conceicao EP, Sim XYJ, Aung MK, Tan KY, Wong HM, Wijaya L, Tan BH, Ling MLVI (2020) Minimising intra-hospital transmission of COVID-19: the role of social distancing. J Hosp Infect 105(2):113-115
6. Mavrogenis AF, Quaile A, Scarlat MM (2020) The virus crisis affects orthopaedic surgery and scientific activities worldwide. International Orthopaedics (SICOT) 44:813-817

7. Huang C, Wang Y, Li X et al (2020) Clinical features of patients infected with 2019 novel coronavirus in Wuhan, China. Lancet. 395(10223):497-506

8. Vanhems P (2020) Fast nosocomial spread of SARS-CoV2 in a French geriatric unit Lyon Study Group on Covid-19 infection. Infect Control Hosp Epidemiol. https://doi.org/10.1017/ice.2020. 99

9. Foreman KJ, Marquez N, Dolgert A et al (2018) Forecasting life expectancy, years of life lost, and all-cause and cause-specific mortality for 250 causes of death : reference and alternative scenarios for 2016-40 for 195 countries and territories. Lancet. 392(10159): 2052-2090

10. Núñez JH, Sallent A, Lakhani K et al (2020) Impact of the COVID19 pandemic on an emergency traumatology service: experience at a tertiary trauma centre in Spain. Injury. 51(7):1414-1418

11. Meng Y, Leng K, Shan L et al (2020) A clinical pathway for preoperative screening of COVID-19 and its influence on clinical outcome in patients with traumatic fractures. International Orthopaedics (SICOT) 44:1549-1555

12. Verity R, Okell LC, Dorigatti I, Winskill P, Whittaker C, Imai N, Cuomo-Dannenburg G, Thompson $\mathrm{H}$ et al (2020) Estimates of the severity of coronavirus disease 2019: a model-based analysis. Lancet Infect Dis 20(6):669-677

13. Parvizi J, Gehrke T, Krueger CA et al (2020) Resuming elective orthopaedic surgery during the COVID-19 pandemic. J Bone Jt Surg. https://doi.org/10.2106/jbjs.20.00844

14. Shah A, Memon M, Kay J, Wood TJ, Tushinski DM, Khanna V (2019) Preoperative patient factors affecting length of stay following total knee arthroplasty: a systematic review and meta-analysis. J Arthroplast 34(9):2124-2165

15. Piuzzi NS, Strnad GJ, Ali Sakr Esa W et al (2019) The main predictors of length of stay after total knee arthroplasty. J Bone Jt Surg. 101(12):1093-1101

16. Sutton JC, Antoniou J, Epure LM, Huk OL, Zukor DJ, Bergeron SG (2016) Hospital discharge within 2 days following total hip or knee arthroplasty does not increase major-complication and readmission rates. J Bone Jt Surg. 98(17):1419-1428

17. Pollock M, Somerville L, Firth A, Lanting B (2016) Outpatient total hip arthroplasty, total knee arthroplasty, and unicompartmental knee arthroplasty: a systematic review of the literature. JBJS Rev. https://doi.org/10.2106/JBJS.RVW.16.00002

18. Sanclemente-Boli T, Ponce-Ruiz S, Álvarez-Lorenzo C et al (2019) Effectiveness of a multidisciplinary educational intervention in patients with hip fracture: SWEET HOME study. Med Clin (Barc) 153(12):446-453

19. Gonçalves-Bradley D, Iliffe S, Doll H et al (2017) Early discharge hospital at home. Cochrane Database Syst Rev. https://doi.org/10. 1002/14651858.CD000356.pub4

Publisher's note Springer Nature remains neutral with regard to jurisdictional claims in published maps and institutional affiliations. 